\title{
Distribution of Ethno Medicinal Plants Along Some Important Roads: A Case Study of Northern Mizoram
}

\author{
Samar Kumar Banerjee ${ }^{1}$, Mousumi Banerjee ${ }^{2}$, Anjani Kumar Srivastava ${ }^{1}$ \\ ${ }^{1}$ Department of Botany, Ranchi University, Ranchi, India \\ ${ }^{2}$ Department of Botany, Faculty of Post Graduate Studies, Scottish Church College, Kolkata, India
}

Email address:

samarbanerjeel@gmail.com (S. K. Banerjee), mousumibanerjee.botany@gmail.com (M. Banerjee), anjani.ranjana@gmail.com (A. K. Srivastava)

\section{To cite this article:}

Samar Kumar Banerjee, Mousumi Banerjee, Anjani Kumar Srivastava. Distribution of Ethno Medicinal Plants Along Some Important Roads: A Case Study of Northern Mizoram. American Journal of Plant Biology. Vol. 4, No. 2, 2019, pp. 16-23.

doi: 10.11648/j.ajpb.20190402.12

Received: August 1, 2019; Accepted: September 17, 2019; Published: September 30, 2019

\begin{abstract}
Mizoram state of India is part of the biodiversity hotspots of the world, the Eastern Himalayan biodiversity hotspot of South Asia which shows roadside rich diversity of flora. These floras are one of the source of carbon sink along the road and also source of medicinal resources for local villagers. India is world leader in the era of medical pluralism because it has strong evidence based biomedical sciences, as well as an immensely rich indigenous medical heritage of its own. Some works related to medicinal plants have been reported by some researchers in some districts and localized area in Mizoram. Till date no work has been reported on the diversity and ecology of medicinal flora growing along the Hill Roads in Mizoram. For listing of the medicinal plants along the roads four (4) major roads of northern part of Mizoram in three different locations were selected for the present study which were surveyed with team of experts for two consecutive years during the months of October to January (2017 \& 2018) within 30m of the roads by traversing on foot on both side of the roads. Data on uses of the plants were collected through questionnaire, interviews and discussions with local people local Vadhya and old men and women community along the roads. During survey 318 traditional medicinal plants were recorded, out of which 170 are trees, 48 are shrubs and 100 are herbs. Status assessments of the plants indicate the fact that very few plants has been listed in IUCN list. Study reveals that distribution of these plants are being rare and endangered in their natural habitat due to several factors which need to be conserved and cultivated for their perpetual existence. Proper attention should be made during further capacity augmentation of these roads for conservation of these medicinal plants. The paper enlists the current diversity, habitat and ecology of the ethno medicinal plants and impact of road development on the flora along the road. The study is likely to help in further capacity augmentation/widening of these roads without harming the current diversity of the medicinal flora growing along the road.
\end{abstract}

Keywords: Hill Roads, Diversity, Ecology, Hotspots, Conservation, Medicinal Plants

\section{Introduction}

The current global health sector trends suggest that medical pluralism, to which Indian traditional medical systems can contribute critically, will shape the future of health care. This shift from singularity to plurality is taking place because it is becoming increasingly evident that no single source of health science has the capacity to contribute solutions to all of societies health needs. India has a comparative advantage to be a world leader in the era of medical pluralism because it has strong foundations in evidence based biomedical sciences, as well as an immensely rich and complex indigenous medical heritage of its own [1].

According to the World Health organization (WHO) as many as $80 \%$ of world's population depends today on traditional medicine for their primary health care needs. The practice of ethno medicine is an important vehicle for understanding indigenous societies and their relationships with nature [2]. In recent decade significant changes occurring within several aspects of ethno medicine as a result of environmental degradation and tremendous changes in 
modern, social, and economic systems [2]. These factors in totality resulted in disappearance of ethno medicinal plants at regional as well as global scale [3].

The Eastern Himalayas is hottest of the 34 biodiversity hotspots of the world. It comprises of a mountain range in South Asia which is youngest of all mountain ranges existing on the face of the earth. It is still in an evolving state. The ecosystem of the region, therefore, naturally exhibit great dynamism. It also holds great significance from ecological and evolutionary point of view. This region is rich in biodiversity and harbours largest number of endemics and Schedule I species as compared to any other part of India [4]

Mizoram, in North -East of India, is a part of Eastern Himalayan biodiversity hotspot, region. Mizoram possesses a geologically distinctive terrain in with low but steep hills ranging in height from $900-1,100$ meters. The hills of the eastern side are slightly higher than their western counterparts. Its unique location, topography with hills and valleys, and geology provide immense ranges of microclimatic conditions which support diverse gene pools of a variety of flora and fauna, making it a "biodiversity rich" area [5]. The study area is also rich in medicinal important plants. About 500 species under 383 genera have been recorded from the state, which has medicinal and ethno botanical uses [6].

The ethno medicinal plants of Mizoram have been reported by various researchers. Darlianthanga (1989) who reported medicinal plants used for the treatment of 97 diseases [7]; Saptawna (1990) reported 58 species Lallianthanga (1990) reported 128 plant species [8, 9], Vailinga (1991) documented 165 diseases and their ethno medicines [10] and Chawngkunga (1996) documented 85 ethno medicinal plants [11]. Some other notable contributions were made by Lalramnghinglova and Jha (1997) and Lalnundanga et al. (1997) [12, 13]. Lalramnghinglova (2003) documented 126 ethno medicinal plants [12]. Rai \& Lalramnghinglova reported 57 less known ethno medicinal plant species belonging to 52 genera and 36 families [14]. Lalfakzuala et al (2007) reported that tribal of western Mizoram use 89 plant species as herbal medicine [15]. Lalzarzovi et al (2016) has documented 56 species of medicinal plants belonging to 54 genera and 35 families which are used traditionally within the city of Aizawl [16]. Rama Shankar (2009) reported use of 39 species as medicinal plants by local people in Kolasib, Aizawl, Champhai and Darlawn districts of Mizoram [17]. Laha et al (2016) recoded 53 species of plant distributed over 49 genera and 39 families which are used by local people of Mizoram as medicines for treatment of diabetic [18].

Although we have various workers reporting the ethno medicinal plant found in the state of Mizoram, no study have been reported on medicinal flora along the roads in Mizoram and impact of road development on these plants.

This study aims at documenting the medicinal plants which are found along important roads of Northern part of Mizoram which have been proposed for capacity augmentation and also deals with the impact of capacity augmentation of the roads on medicinal flora along the roads.

\section{Materials and Methods}

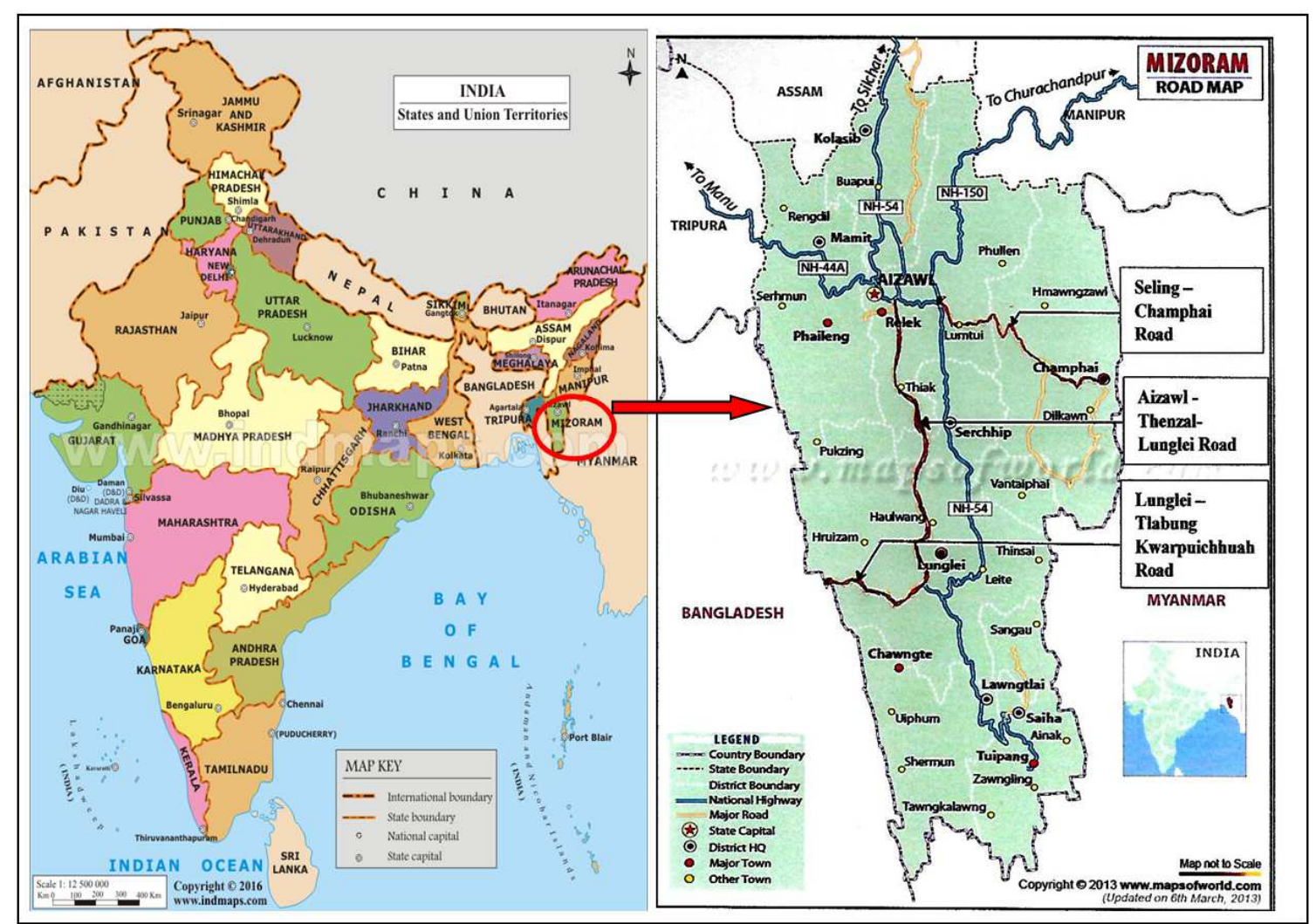

Figure 1. Roads considered for study. 
For listing of the medicinal plants along the roads four (4) major roads of northern part of Mizoram in three different locations were selected for the present study. Selected roads were - NH-44A road (Length $=55 \mathrm{Km})$, Serchip - Thenzal road $($ Length $=27 \mathrm{Km}), \mathrm{NH}-54$ road $($ Length $=56 \mathrm{Km})$ and Champhai - Zokhwatar road $(26 \mathrm{~km})$ (Figure 1). All the roads were surveyed with team of experts for two consecutive years during the months of October to January (2017 \& 2018). Data were collected within $30 \mathrm{~m}$ (considering corridor of impact, COI) of the roads by traversing on foot on both side of the roads (valley side and hill side $15 \mathrm{~m}$ each). Identification of plants and the data on the uses of the plants were collected through questionnaire, interviews and discussions among local people in presence of the Village Council Presidents (VCP), local Vadhya and old men and women of local village community along the roads.

Several visits were made to the field with these resource persons who helped in identifying and naming of some of these plants and their medicinal uses. It consists of basic documentation of traditional botanical knowledge of the local people through interaction with them. Some very common plants like well-known trees were not collected for voucher specimens. Only information provided by the local people for these species was recorded. Specimens were collected and processed into mounted herbarium sheets and were identified using different literatures [19]. Voucher specimens were deposited into the Herbarium of the University Department of Botany Ranchi University, Ranchi.

\section{Results}

This study documented 33 species of medicinal important plants distributed over 21 families along COI of NH-44 A which is $55 \mathrm{~km}$ in length. Plants of Verbanaceae family were found dominant family. During survey 318 traditional medicinal plants were recorded, out of which 170 are trees, 48 are shrubs and 100 are herbs (Figures $2 \& 3$ ). Status assessments of the plants indicate the fact that Bauhinia variegata (L.) has been listed as list concern in IUCN red list version 3.1. (Table 1)

Gleditsia assamica Bor. has been listed as vulnerable in IUCN red list version 3.1. G. assamica grows in primary and secondary forests of Northeast India at an elevation range of 100-250 m altitude. It is a medium to large sized tree (15-20 $\mathrm{m})$ with a conical crown and thick canopy. Pods have ethno botanical use and Garo tribes of Nokrek Biosphere Reserve use the paste to heal stomachache. (Table 1)

Clear felling along with habitat degradation severely affected the natural population of G. assamica and the species is also listed as 'Vulnerable' in the IUCN red list of threatened species (IUCN 2009).

Other plants neither listed in IUCN red list version 3.1 nor in Catalogue of Life (COL), 2018. (Refer Table 1)

Table 1. List of Medicinal plants in proposed ROW of NH-44A road (Length $55 \mathrm{~km}$ ).

\begin{tabular}{|c|c|c|c|c|c|c|}
\hline \multirow{2}{*}{ Sl. No } & \multicolumn{2}{|l|}{ Name of Plant species } & \multirow{2}{*}{ Family } & \multirow{2}{*}{ Part used } & \multirow{2}{*}{$\begin{array}{l}\text { Total No. of } \\
\text { individuals }\end{array}$} & \multirow{2}{*}{$\begin{array}{l}\text { Status as per IUCN } \\
\text { RED list } 2017 \text { and COL }\end{array}$} \\
\hline & Scientific Name & Local Name & & & & \\
\hline \multicolumn{7}{|l|}{ Herbs } \\
\hline 1. & Desmodium sequax Wall. & Chabet & Leguminosae & Leaf & 45.0 & Not listed \\
\hline 2. & Ageratum conyzoides (L.) L. & Vaihlenhlo & Asteraceae & Whole body & 20.0 & Not listed \\
\hline 3. & Solanum nigrum L. & An-hling & Solanaceae & Fruit & 3.0 & Not listed \\
\hline 4. & Scoparia dulcis L. & Perhpawngchaw & Scrophulariaceae & Leaf, Stem, Root & 32.0 & Not listed \\
\hline \multicolumn{7}{|l|}{ Shrubs } \\
\hline 5. & Curcuma longa $\mathrm{L}$. & Ai-eng & Zingiberaceae & Whole body & 7.0 & Not listed \\
\hline 6. & $\begin{array}{l}\text { Homalomena aromatic (Spreng.) } \\
\text { Schott }\end{array}$ & Anchiri & Araceae & Root & 5.0 & Not listed \\
\hline 8. & Clerodendrum infortunatum $\mathrm{L}$. & Phuihnamchhia & Verbenaceae & Root, Leaves & 8.0 & Not listed \\
\hline 9. & Sarcandra glabra (Thunb.) Nakai & Sen-thet & Verbenaceae & Root, Leaves & 6.0 & Not listed \\
\hline & Manihotesculenta Crantz & Pangbal & Euphobiaceae & Root & 5.0 & Not listed \\
\hline \multicolumn{7}{|l|}{ Trees } \\
\hline 14. & Bauhinia variegata $\mathrm{L}$. & Vaube & Caesalpiniaceae & Bark, Leaves & 12.0 & Least concern ver3.1 \\
\hline 15. & $\begin{array}{l}\text { Grevillea robusta A. Cunn. ex R. } \\
\text { Br. }\end{array}$ & Silver oak & Proteacae & & 3.0 & Not listed \\
\hline 16. & Areca catechu L. & Kuhva kung & Arecaceae & Seeds & 38.0 & Not listed \\
\hline 17. & Litsea monopetala (Roxb.) Pers. & Nauthak & Lauraceae & Root, Bark, Leaf & 7.0 & Not listed \\
\hline 18. & Derris robusta (DC.) Benth & Thingkha & Fabaceae & Bark & 6.0 & Not listed \\
\hline 19. & Garcinia lanceifolia Roxb. & Chengkek & Clusiaceae & Fruits, Leaves & 7.0 & Not listed \\
\hline 20. & Artocarpus heterophyllus Lam. & Lamkhuang & Moraceae & Root & 4.0 & Not listed \\
\hline 21. & $\begin{array}{l}\text { Gmelina arborea } \\
\text { Roxb. }\end{array}$ & Thlanvawng & Verbenaceae & $\begin{array}{l}\text { Root, Leaf, Flower, } \\
\text { Fruits }\end{array}$ & 8.0 & Not listed \\
\hline 22. & Schima wallichii Choisy & Khiang & Theaceae & Fruits, Bark & 22.0 & Not listed \\
\hline 23. & Duabanga grandiflora (DC.) Walp. & Zuang & Lythraceae & Bark & 2.0 & Not listed \\
\hline 24. & Callicarpa arborea Roxb. & Hnahkiah & Verbenaceae & Bark, Leaf & 4.0 & Not listed \\
\hline 25. & $\begin{array}{l}\text { Heteropanax fragrans (Roxb.) } \\
\text { Seem. }\end{array}$ & Chang-khen & Araliaceae & Root, Bark & 5.0 & Not listed \\
\hline 26. & Castanopsis tribuloides (Sm.) A. & Thingsia & Fagaceae & Stem & 9.0 & Not listed \\
\hline
\end{tabular}




\begin{tabular}{|c|c|c|c|c|c|c|}
\hline \multirow{2}{*}{ SI. No } & \multicolumn{2}{|l|}{ Name of Plant species } & \multirow{2}{*}{ Family } & \multirow{2}{*}{ Part used } & \multirow{2}{*}{$\begin{array}{l}\text { Total No. of } \\
\text { individuals }\end{array}$} & \multirow{2}{*}{$\begin{array}{l}\text { Status as per IUCN } \\
\text { RED list } 2017 \text { and COL }\end{array}$} \\
\hline & Scientific Name & Local Name & & & & \\
\hline & DC. & & & & & \\
\hline 27. & Gleditsia assamica Bor & Hluk-ral & Leguminsae & Seed, bark, Fruit & 3.0 & Vulnerable $\mathrm{B} 1+2 \mathrm{c}$ ver 2.3 \\
\hline 28. & Syzygium cumini (L.) Skeels. & Len-hmui & Myrtaceae & Seed, bark, Fruit & 3.0 & Not listed \\
\hline 29. & $\begin{array}{l}\text { Aporosa octandra (Buchanan- } \\
\text { Hamilton ex D. Don) Vickery }\end{array}$ & Chhawntual & Phyllanthaceae & Bark & 8.0 & Not listed \\
\hline 30. & Сinnaтотит verum J. Presl & Thakthing & Lauraceae & $\begin{array}{l}\text { Stem bark and root } \\
\text { bark }\end{array}$ & 6.0 & Not listed \\
\hline 31. & Mesua ferrea $\mathrm{L}$. & Herhse. & Clusiaceae & Fruits \& flower & 4.0 & Not listed \\
\hline 32. & Tectona grandis L. f. & Teak & Verbenaceae & Root, Bark, flower & 10.0 & Not listed \\
\hline 33. & $\begin{array}{l}\text { Persea odoratissima (Nees) } \\
\text { Kosterm }\end{array}$ & Bulfek & Luraceae & Root, Bark, Flower & 3.0 & Not listed \\
\hline
\end{tabular}

Total plants to be cut $=318$, trees $=170$, shrub $=48$, Herb $=100$

In Serchip - Thenzal road which is $27 \mathrm{Km}$ long, 29 species of medicinal plants distributed over 22 families were recorded. During survey 152 traditional medicinal plants recorded in COI out of which 79 are trees, 19 are shrubs and 54 are herbs (Figures $2 \& 3$ ). Status assessments of the plants indicate the fact that Bauhinia variegata (L.) and Tamarindus indica $\mathrm{L}$ has been listed as list concern in IUCN red list version 3.1. Other plants neither listed in IUCN red list version 3.1 nor in Catalogue of Life (COL), 2018. (Refer Table 2)

Table 2. List of Medicinal Plants with in Proposed Right of way of the Serchip - Thenzal road (Length $=27 \mathrm{Km}$ ).

\begin{tabular}{|c|c|c|c|c|c|c|}
\hline \multirow{2}{*}{$\begin{array}{l}\text { Sl. } \\
\text { No }\end{array}$} & \multicolumn{2}{|l|}{ Name of plant species } & \multirow{2}{*}{ Family } & \multirow{2}{*}{ Part used } & \multirow{2}{*}{$\begin{array}{l}\text { Total No. of } \\
\text { individuals }\end{array}$} & \multirow{2}{*}{$\begin{array}{l}\text { Status as per IUCN RED } \\
\text { list } 2017 \text { and COL }\end{array}$} \\
\hline & Scientific Name & Local Name & & & & \\
\hline \multicolumn{7}{|c|}{ Herb } \\
\hline 1 & $\begin{array}{l}\text { Alternanthera philoxeroides (Mart.) } \\
\text { Griseb. }\end{array}$ & Nghateril & Amaranthaceae & Extract & 3.0 & Not listed \\
\hline 2 & Ageratum conyzoides (L.) L. & Vailenhlo & Asteraceae & Whole body & 11.0 & Not listed \\
\hline 3 & $\begin{array}{l}\text { Chromolaena odorata (L.) King \& } \\
\text { Robinson }\end{array}$ & Tlangsam & Asteraceae & Leaf, Stem, Seed & 12.0 & Not listed \\
\hline 5 & Desmodium sequax Wall. & Chabet & Leguminosae & Leaf & 4.0 & Not listed \\
\hline 6 & Andrographis paniculata Nees. & Hnahkhapui & Acanthaceae & Leaves, stem, root & 20.0 & Not listed \\
\hline \multicolumn{7}{|c|}{ Shrubs } \\
\hline 7 & Lantana camara L. & $\begin{array}{l}\text { Shillong } \\
\text { tlangsam }\end{array}$ & Verbanaceae & Whole body & 9.0 & Not listed \\
\hline 8 & Hibiscus sabdariffa $\mathrm{L}$. & An-thur & Malvaceae & Leaves Fruits, Flower & 3.0 & Not listed \\
\hline 9 & Sidaacuta Burm. f. & Khing-khih & Malvaceae & Root, Leaves & 2.0 & Not listed \\
\hline 11 & Bauhinia variegate L. & Vaube & Caesalpiniaceae & Bark, Leaf & 5.0 & Least concern ver 3.1 \\
\hline 12 & Adhatoda vasica Nees. & Kawldawi & Acanthaceae & Leaf, root & 8.0 & Not listed \\
\hline 13 & Curcuma longa $\mathrm{L}$. & Ai-eng & Zingiberaceae & Whole body & 7.0 & Not listed \\
\hline 14 & Clerodendrum infortunatum $\mathrm{L}$. & Phuihnamchhia & Verbenaceae & Root, Leaves & 8.0 & Not listed \\
\hline 15 & Manihot esculenta Crantz & Pangbal & Euphobiaceae & Root & 5.0 & Not listed \\
\hline 16 & Asparagus recemosus Willd. & Arkebawk & Liliaceae & Rhizome & 9.0 & Not listed \\
\hline \multicolumn{7}{|c|}{ Trees } \\
\hline 17 & $\begin{array}{l}\text { Aporosa octandra (Buchanan-Hamilton } \\
\text { ex D. Don) }\end{array}$ & Chhawntual & Phyllanthaceae & Bark. & 4.0 & Not listed \\
\hline 18 & $\begin{array}{l}\text { Cinnamomum tamala (Buchanan- } \\
\text { Hamilton) T. Nees \& Ebermaier }\end{array}$ & $\begin{array}{l}\text { Tespata, } \\
\text { Hnahrimtui }\end{array}$ & Lauraceae & $\begin{array}{l}\text { Stem Bark and root } \\
\text { bark. }\end{array}$ & 6.0 & Not listed \\
\hline 19 & Cinnamomum verum J. Presl & Thakthing & Lauraceae & $\begin{array}{l}\text { Root bark, stem bark, } \\
\text { leaves }\end{array}$ & 4.0 & Not listed \\
\hline 20 & Phyllanthus emblica Linnaeus & Sunhlu & Phyllanthaceae & Bark, fruit. & 7.0 & Not listed \\
\hline 21 & Eucalyptus globulus Labillardière & Eucalyptus & Myrtaceae & Leaves & 5.0 & Not listed \\
\hline 22 & Mesua ferrea Linnaeus & Herhse & Clusiaceae & Fruit, flower. & 3.0 & Not listed \\
\hline 24 & Terminalia chebula Retzius & Reraw & Combretaceae & Fruits & 7.0 & Not listed \\
\hline 25 & Aquilaria malaccensis Lamarck & Thingrai. & Thymelaeaceae & Stem bark & 6.0 & Not listed \\
\hline 26 & Butea frondosa Koen. ex. Roxb. & Tuahpui & Papilionaceae & $\begin{array}{l}\text { Leaf, flower, } \\
\text { seed }\end{array}$ & 11.0 & Not listed \\
\hline 27 & Vitex peduncularis Wall. & Thingkhawi-Hlu & Verbenaceae & leaf, root and bark & 17.0 & Not listed \\
\hline 28 & Heteropanax fragrans (Roxb.) Seem. & Chang-khen & Araliaceae & Root, Bark & 5.0 & Not listed \\
\hline 29 & Tamarindus indica $\mathrm{L}$. & Chhimakelek & Caesalpiniaceae & seed & 2.0 & Least Concern ver 3.1 \\
\hline
\end{tabular}

Total plants to be cut $=152$. Trees 79, shrubs $=19$, Herb $=54$ 
This study also documented 32 species of medicinal important plants distributed over 25 families along COI of NH-54 which is $56 \mathrm{~km}$ in length. Plants of Aracaceae and Fabaceae family were found dominant family. During survey 239 traditional medicinal plants were recorded, out of which 126 are trees, 31 are shrubs and 82 are herbs. Status assessments of the plants indicate the fact that Caryota urens L. and Aeschynomene indica L. has been listed as list concern in IUCN red list version 3.1. Other plants neither listed in IUCN red list version 3.1 nor in Catalogue of Life (COL), 2018. (Refer Table 3)

Table 3. List of Medicinal Plants with in Proposed Right of way of the NH-54 road (Length $=56 \mathrm{Km}$ ).

\begin{tabular}{|c|c|c|c|c|c|c|}
\hline \multicolumn{3}{|c|}{ Name of Plant species } & \multirow{2}{*}{ Family } & \multirow{2}{*}{ Part used } & \multirow{2}{*}{$\begin{array}{l}\text { Total No. of } \\
\text { individuals }\end{array}$} & \multirow{2}{*}{$\begin{array}{l}\text { Status as per IUCN } \\
\text { RED list } 2017 \text { and COL }\end{array}$} \\
\hline Sl. No. & Scientific Name & Local Name & & & & \\
\hline \multicolumn{7}{|l|}{ Herbs } \\
\hline 1 & Desmodium sequax Wall. & Chabet & Fabaceae & Leaf & 8.0 & Not listed \\
\hline 2 & Scoparia dulcis L. & Perhpawngchaw & Scrophulariaceae & Whole plant & 25.0 & Not listed \\
\hline 3 & Lantana camara $\mathrm{L}$. & Shillong & Verbanaceae & Leaf & 7.0 & Not listed \\
\hline 4 & Curcuma longa $\mathrm{L}$. & Tlangsam & Zingiberaceae & Root & 8.0 & Not listed \\
\hline 5 & $\begin{array}{l}\text { Homalomena aromatic (Spreng.) } \\
\text { Schott }\end{array}$ & Ai-eng & Araceae & Bark & 3.0 & Not listed \\
\hline 6 & Solanum nigrum L. & An-hling & Solanaceae & Leaf and fruits & 11.0 & Not listed \\
\hline 7 & Hibiscus sabdariffa $L$. & An-thur & Malvaceae & Fruit \& flower & 4.0 & Not listed \\
\hline 8 & Ageratum conizoides (L.) L. & Vailenhlo & Asteraceae & Whole plant & 16.0 & Not listed \\
\hline \multicolumn{7}{|l|}{ Shrubs } \\
\hline 9 & Caryota urens L. & Tum (Palm Tree) & Aracaceae & Stem & 7.0 & Least Concern ver 3.1 \\
\hline 10 & Aeschynomene indica L. & Hlo-Nuar-Suak & Fabaceae & Root, leaf and flower & 5.0 & Least Concern ver 3.1 \\
\hline 11 & $\begin{array}{l}\text { Ammomum maximum Roxb. } \\
\text { Tabernaemontana divaricate }\end{array}$ & Aidu & Zingiberaceae & Stem, buds & 6.0 & Not listed \\
\hline 13 & Garcinia lancifolia Roxb. & Pelh & Clusiaceae & Fruits & 4.0 & Not listed \\
\hline \multicolumn{7}{|l|}{ Trees } \\
\hline 14 & Tectona grandis L. f. & Teak & Verbenaceae & Root, Bark, flower & 11.0 & Not listed \\
\hline 15 & Alstonia scholaris (L.) R. Br. & Thuamriat & Apocynaceae & Bark & 6.0 & Not listed \\
\hline 16 & Bauhinia variegate L. & Vaube & Caesalpiniaceae & Bark and Leaf & 8.0 & Not listed \\
\hline 17 & Areca catechu L. & Kuhva kung & Arecaceae & Seeds & 23.0 & Not listed \\
\hline 18 & Garcinia lanceifolia Roxb. & Chengkek & Clusiaceae & Frits and Leaves & 4.0 & Not listed \\
\hline 19 & Artocarpus heterophyllus Lam. & Lamkhuang & Moraceae & Root & 6.0 & Not listed \\
\hline 20 & Erythrina stricta Roxb. & Fartuah & Fabaceae & Bark and Leaf & 5.0 & Not listed \\
\hline 21 & Gmelina arborea Roxb. & Thlanvawng & Verbenaceae & Root, flower, & 5.0 & Not listed \\
\hline 22 & Schima wallichii choisy. & Khiang & Theaceae & Fruit, Bark & 5.0 & Not listed \\
\hline 23 & Duabanga grandiflora (DC.) Walp. & Zuang & Sonneratiaceae & Bark & 2.0 & Not listed \\
\hline 24 & Callicarpa arborea Roxb. & Hnahkiah & Verbenaceae & Bark & 2.0 & Not listed \\
\hline 25 & Castanopsis tribuloides (Sm.) A. DC. & Thingsia & Fagaceae & Stem & 2.0 & Not listed \\
\hline 28 & Parkia timoriana (DC.) Merr. & Zawngtah & Mimosaceae & Fruit, root, leaf & 4.0 & Not listed \\
\hline 28 & Drimycorpus racemosus & Vawmbal & Anacardiaceae & Bark & 5.0 & Not listed \\
\hline 30 & Terminalia chebula Retzius. & Reraw & Combretaceae & Fruit & 6.0 & Not listed \\
\hline 31 & Oroxylum indicum (Linnaeus) Kurz & Archangkawm & Bignoniaceae & $\begin{array}{l}\text { Stem bark and root } \\
\text { bark }\end{array}$ & 3.0 & Not listed \\
\hline 32 & Ziziphus jujube Mill. & Bawrai & Rhamnaceae & Fruit, Bark, root, leaf & 12.0 & Not listed \\
\hline
\end{tabular}

Total plants to be cut $=239$. Trees $=126$, Shrub $=31$ and Herb $=82$

In $\mathrm{C}-\mathrm{Z}$ road which is $26 \mathrm{Km}$ long, 25 species of medicinal plants distributed over 20 families were recorded. During survey 160 traditional medicinal plants recorded in COI out of which 42 are trees, 57 are shrubs and 61 are herbs (Figures $2 \& 3$ ). Status assessments of the plants indicate the fact that only Tamarindus indica L. has been listed as list concern in IUCN red list version 3.1. Other plants neither listed in IUCN red list version 3.1 nor in Catalogue of Life (COL), 2018. (Refer Table 4)

Table 4. List of Medicinal plants in proposed ROW of C-Z road (Length $26 \mathrm{~km}$ ).

\begin{tabular}{|c|c|c|c|c|c|c|}
\hline \multirow{2}{*}{ Sl. No. } & \multicolumn{2}{|l|}{ Name of Plant species } & \multirow{2}{*}{ Family } & \multirow{2}{*}{ Part used } & \multirow{2}{*}{$\begin{array}{l}\text { Total No. of } \\
\text { individuals }\end{array}$} & \multirow{2}{*}{$\begin{array}{l}\text { Status as per IUCN } \\
\text { RED list } 2017 \text { and COL }\end{array}$} \\
\hline & Scientific Name & Local Name & & & & \\
\hline \multicolumn{7}{|l|}{ Herbs } \\
\hline 1 & Mikania micrantha Kunth. & Japanhlo & Asteraceae & Leaf & 6.0 & Not listed. \\
\hline 2 & Ageratum conizoides (L.) L. & Vailenhlo & Asteraceae & Whole plant & 25.0 & Not listed \\
\hline 3 & $\begin{array}{l}\text { Chromolaena odorata (L.) R. M. } \\
\text { King \& H. Rob. }\end{array}$ & Tlangsam & Asteraceae & Leaf, Stem, Seed & 4.0 & Not lissted \\
\hline
\end{tabular}




\begin{tabular}{|c|c|c|c|c|c|c|}
\hline \multirow{2}{*}{ SI. No. } & \multicolumn{2}{|l|}{ Name of Plant species } & \multirow{2}{*}{ Family } & \multirow{2}{*}{ Part used } & \multirow{2}{*}{$\begin{array}{l}\text { Total No. of } \\
\text { individuals }\end{array}$} & \multirow{2}{*}{$\begin{array}{l}\text { Status as per IUCN } \\
\text { RED list } 2017 \text { and COL }\end{array}$} \\
\hline & Scientific Name & Local Name & & & & \\
\hline 4 & Urena lobate L. & Sehnap & Malvaceae & Leaf, Root, Bark & 12.0 & Not listed \\
\hline 5 & Morus alba L. & Theihmu (Hlingnei) & Moraceae & Fruit, Bark & 7.0 & Not listed \\
\hline 6 & Mallotus roxburghianus Mull. Arg. & Zawngtenawhlung & Euphorbiaceae & Bark, Leaves & 7.0 & Not listed \\
\hline \multicolumn{7}{|l|}{ Shrubs } \\
\hline 7 & $\begin{array}{l}\text { Viburnum mullaha Buch.-Ham. ex. } \\
\text { D. Don. }\end{array}$ & Vawngser & Stemonaceae & Root & 2.0 & Not listed \\
\hline 8 & Derris robusta (DC.) Benth. & Thingkha & Fabaceae & Bark & 21.0 & Not listed \\
\hline 9 & Schima wallichii Choisy & Khiang & Theaceae & Bark, Fruit & 10.0 & Not listed \\
\hline 10 & Callicarpa arborea Roxb. & Hnahkiah & Verbenaceae & Bark, Leaf & 4.0 & Not listed \\
\hline 11 & Bauhinia variegate $\mathrm{L}$. & Vaube & Caesalpiniaceae & Bark, Leaf & 5.0 & Not listed \\
\hline 12 & Adhatoda vasica Nees. & Kawldawi & Acanthaceae & Leaf, root & 8.0 & Not listed \\
\hline 13 & Curcuma longa $\mathrm{L}$. & Ai-eng & Zingiberaceae & Whole body & 7.0 & Not listed \\
\hline \multicolumn{7}{|l|}{ Trees } \\
\hline 14 & Albizia lebbeck (L.) Benth & Thingri & Mimosaceae & Bark & 5.0 & Not listed \\
\hline 15 & Emblica offinalis Gaertn. & Sunhlu & Phyllanthaceae & Fruit, Bark, Root & 7.0 & Not listed \\
\hline 16 & Azadirachta indica A. Juss. & Neem & Meliaceae & Bark, Leaf, Fruit & 6.0 & Not listed \\
\hline 17 & Albizia chinensis (Osbeck) Merr. & Vang & Mimosaceae & Bark & 4.0 & Not listed \\
\hline 18 & Anogeissus acuminate (Rox. ex DC.) & Zairum & Combretaceae & Bark, Leaf & 5.0 & Not listed \\
\hline 19 & Ficus religiosa $\mathrm{L}$. & Bung & Moraceae & Bark, root, fruits & 4.0 & Not listed \\
\hline 20 & Tamarindus indica $\mathrm{L}$. & Chhimakelek & Caesalpiniaceae & seed & 2.0 & Least Concern ver 3.1 \\
\hline 21 & Annona squamosa L. & Theiarbawn & Annonaceae. & Leaf, root, fruit, seed & 4.0 & Not listed \\
\hline 22 & Vitex peduncularis Wall. & Thingkhawi-Hlu & Verbenaceae & leaf, root and bark & 3.0 & Not listed \\
\hline 23 & Anthocephalus cadamba Miq. & Banphar & Rubiaceae & Bark, leaf & 7.0 & Not listed \\
\hline 24 & Butea frondosa Koen. ex. Roxb. & Tuahpui & Papilionaceae & Leaf, flower, seed & 6.0 & Not listed \\
\hline 25 & Artocarpus chaplasha Roxb. & tatkawng & Moraceae. & Bark & 3.0 & No listed \\
\hline
\end{tabular}

Plants to be cut $=160$. Trees $=42$, Shrub $=57$ and Herbs $=61$

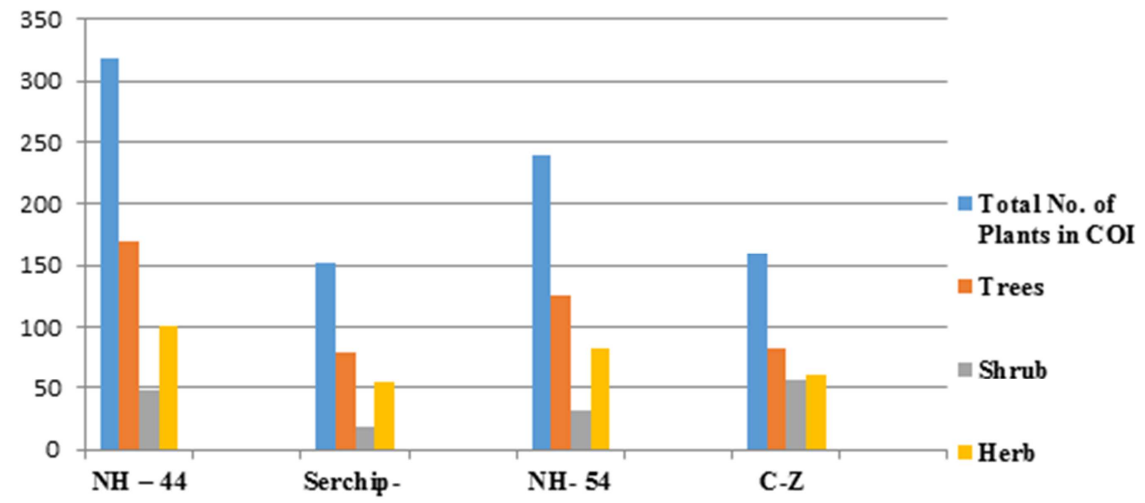

Figure 2. Distribution of medicinal plants along the roads.

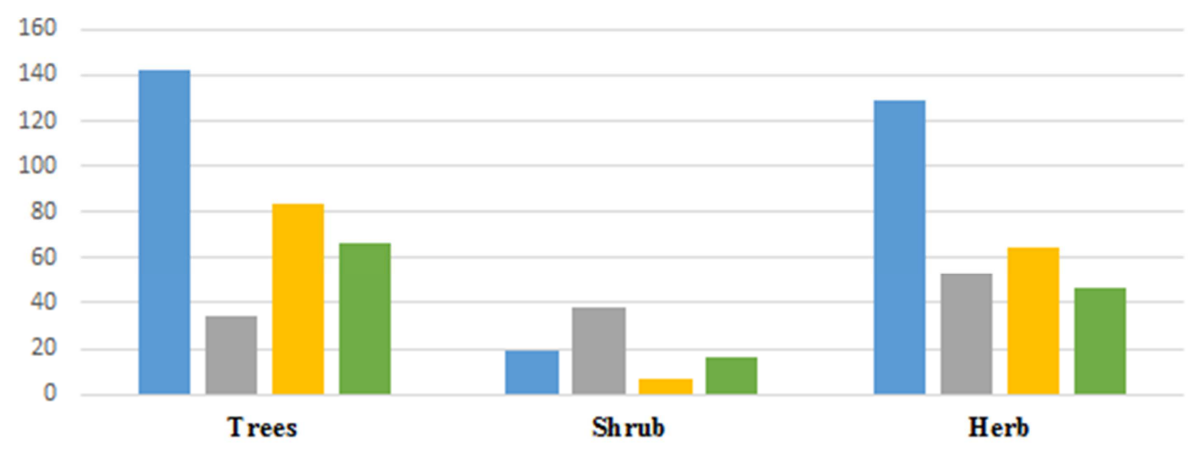

$\square$ NH - 44 A $\quad$ Serchip-Thenzal road $\quad$ NH- $54 \quad \square$ C-Z ROAD

Figure 3. Habitat wise distribution of medicinal plants.

\section{Discussion}

This study has focused on $164 \mathrm{~km}$ of road on northern part of Mizoram state in India which has been proposed for widening for capacity augmentation of the four (04) roads by the by the state government.

This study has documented 869 species of medicinal plants 
distributed in 32 families and 42 genera which are used traditionally by the local people living in the settlements along the project roads under study. Dominant families with traditional medicinal plants recoded in the project roads belongs to Verbanaceae, Aracaceae, Fabaceae, Scrophlurariace, Theaceae, Asteraceae, Acanthaceae and Malvaceae.

Consultation with local people revealed the fact that different parts of the plants were used for preparation of medicine and among them, leaves were most frequently used (52\% spp.) followed by roots (33\% spp.), bark ( $8 \%$ spp.), whole plant ( $5 \%$ spp.). Other parts used included stem, fruit, flower, rhizome, sap, seed, pith, cladodes, twigs and woodcharcoal ( $2 \%$ spp.).

The mode of preparation varies in different species and the most frequently used modes are decoction and infusion of various plant parts followed by juice of leaves, stem, roots, barks or whole plants. Other modes are paste, powder, raw, steaming. The mode of application may be external or internal. They also confirmed that a single species is used to treat more than one kind of disease and the mode of preparation for the treatment of each disease as well as the plant parts used are different.

It has come out from consultations with local people in the road side villages that these traditional medicinal plants are generally taken for - for fever; crushed leaf juice applied and drunk for inflammatory glands; infusion of leaves taken against tonsillitis some entire plant used in - dysentery and dyspepsia; also used in spleen complaints, colic and strangulation of intestine, constipation, diarrhoea, cholera and bites of rabid jackal. Plant parts are used for stomachache, asthma, leaf juice to stop bleeding, bark decoction taken against colic pain and stomach-ache also applied on wounds and chronic ulcer. Leaf infusion is taken against diarrhoea, bronchitis, asthma, and cancer and liver ailments. Leaves smoked as tobacco for chest complaints and asthma; roasted leaf applied on breast for lump or stony hard breast. Grinded bark mixed with water and used for diabetes. Leaf juice is also used in toothache, taken against pneumonia. Plants are also used for sore-throat, toothache and inflammatory glands, rheumatism, sciatica, wounds and applied internally for eye problems. Use of plants to treat epilepsy, snake bite, urinary and kidney problems, stomachache and piles was also reported by the villagers during consultation.

\section{Conclusion}

Different literatures throughout the world indicate a renewed interest in traditional medicine today. There has been an ever increasing demand for more and more drugs from plant sources during the past decade and this revival of interest is mainly due to the widespread belief that green medicine is safe and more dependable than. many of which have adverse side effects.

According to the World Health organization (WHO) as many as $80 \%$ of world's population depends today on traditional medicine for their primary health care needs. The practice of ethno medicine is an important vehicle for understanding indigenous societies and their relationships with nature.

These medicinal plants are also the source of carbon sink along the road. No work has been reported on the diversity, ecology and utilization of the medicinal plants along the hill roads in Mizoram. These plants are very important for local people living along the roads. Study reveals that distribution of these plants are being rare and endangered in their natural habitat due to several factors which need to be conserved and cultivated for their perpetual existence. A single species may be used to treat more than one kind of disease and the mode of preparation for the treatment of each disease as well as the plant part used may be different. These plants are potential for felling and cutting during new road construction and capacity augmentation of the hill roads. This will not only destroy the eco-system of the area as well as deprive the local people living along the roads from traditional rights of using as traditional medicines and also of the eco-system services.

\section{Recommendation}

Proper attention should be made during further capacity augmentation of these roads for conservation of these medicinal plants. Simultaneously, traditional knowledge should be documented, and preserved for further studies. This type of study would be helpful in monitoring and management of population of the medicinal plants, sustainable road development and eco-efficient and sustainable urban infrastructure approach- initiative for green economic growth in Mizoram.

\section{Author Contributions}

All the three authors are working in team in the project for strategy development of sustainable and green road project. All the three authors are going to field and collecting the samples, identifying the species, preparing herbarium and also interacting with different stake holders. Contributions of all the authors are equal in the research project and preparation of the paper.

\section{Acknowledgements}

Authors are grateful to the Head, University Department of Botany Ranchi University, Ranchi, Prof. A. Mukherjee, Department of Botany, University of Burdwan and Dr. Shampa Bhattacharya, Head Department of Botany, Scottish Church College, Kolkata for their kind inspiration in the present work.

\section{References}

[1] Mukherjee A., Banerjee M, (2014) Modernization of Ayurveda: A brief overview of Indian initiatives. NPC Natural Product Communications, 9, 287-290. 
[2] Anyinam C (1995). Ecology and ethnomedicine: Exploring links between current environmental crisis and indigenous medical practices. Soc. Sci. Med. 40 (3): 321-329.

[3] Baillie JE, Hilton-Taylor C, Stuart SN (eds; 2004) A Global Species Assessment. Gland, Switzerland: The World Conservation Union.

[4] Banerjee Samar Kumar, Banerjee Mousumi and Srivastava Anjani Kumar (2018) Ethnobotanical uses of some Hill road side Pteridophytes in Mizoram, India, Int. J. of. Life Sciences, Volume 6 (3): 761-768.

[5] Swamliana 2013. The book of Mizoram Plants, Published by P. Zakhuma, Chanmari, Aizawl. Mizoram.

[6] Singh N. P, Singh K. P. and Singh, D. K (2002), Flora of Mizoram: volume I: Rununculacae-Asteraceae. Botanical Survey of India.

[7] Darlianthanga, C. 1989. Fa Duthlan Dan leh Mizo Damdawi. Khatla, Aizawl GOI 2011. Census of India, 2011. Registrar General and Census Commissioner of India, Ministry of Home Affairs, New Delhi, India.

[8] Saptawna (1990) Tualchhuak Damdawi Thlan Chhuah: Medicinal Plants \& Selected Traaditional Medicines. Kawlkunga. Tahan. Kalemyo, Pp. 1-216.

[9] Lallianthanga, R. K. 1990. Medicinal Plants of Mizoram (A project report). Mizoram Council.

[10] Vailinga, Rev. M. (1991) Afizo Pipute Sullmu leh Mizo Damdawi. Christian Book Store, Chanmari, Lunglei, pp. 1-27.

[11] Chawngkunga, C. 1996. Tualchhuak Damdawi. Directorate of Health and Family Welfare, Govt. of Mizoram, Aizawl.

[12] Lalramnghinglova, H. 2003. Ethno-Medicinal Plants of Mizoram. Bishen Singh Mahendra Pal Singh, Dehradun, India.

[13] Lalnundanga; Sahoo, U. K. \& Jha, L. K. 1997. Ethnobotanical flora in the humid subtropical semi-evergreen forest of Mizoram. Proceedings National Conference on Healthcare and Developments of Herbal Medicines, Raipur.
[14] K Rai, \& H Lalramnghinglova 2013. Ethnomedicinal plant resources of Mizoram, India: Implication of traditional knowledge in health care system, Ethnobotanical Leaflets 2010 (3), 6.

[15] Lalfakzuahla, R. H., Lalramnghinglova, H., \& Kayeng, H. (2007). Ethnobotanical usage of plants in Western Mizoram, Ind J Trad Knowledge, 6 (3), 486-493.

[16] Lalzarzovi S. T. and Lalramnghinglova H. Traditional use of medicinal plants found within Aizawl city in Mizoram, India, Pleione 10 (2): 269 - 277. 2016. of Science, Technology and Environment, Aizawl, Mizoram.

[17] Rama Shankar, Rawat M. S., Majumder, \& R., Boruah D. (2009). Medico ethno botany of Mizoram (Kolasib, Aizawl, Champhai and Darlawn districts. J Drug Res in Ayur \& Siddha, 30 (3-4), 27-40.

[18] Laha R., Lalthanpuia, Lalmuanpui R., Ralte L and Lalremuruata PC (2016). Indegenous use of antidiabetic plants by ethnic inhabitants of Mizoram, North east India, Journal of Medicinal Plant studies 4 (6): 181-184.

[19] Jain S. K. and Rao R. R. (1977). A Handbook of Field and Herbarium Methods. Today and tomorrow's Printers and Publishers, New Delhi.

[20] A Global Species Assessment. The IUCN Species Survival Commission. The IUCN Species Programme Rue Mauverney 28 CH-1196 Gland, Switzerland p. 217.

[21] Economic survey of Mizoram 2012-2013. Statistical report published by Planning and Program Implementation Department, Government of Mizoram.

[22] India State of Forest report 2015, Forest Survey of India, (Ministry of Environment Forest), Dehradun, page 201 -205.

[23] WHO (2005) National Policy on Traditional Medicine and Regulation of Herbal Medicines - Report of a WHO Global Survey. Geneva.

[24] Kanjilal, P. C; Das. A. \& Purkaystha, C. (eds.), Flora of Assam Vol. 1 (a). Government Press, Shillong, India. 